\title{
PROTOCOLOS UTILIZADOS EM CONSULTÓRIOS, CLÍNICAS E HOSPITAIS VETERINÁRIOS PARA O CONTROLE DE ENDO E ECTOPARASITAS EM PEQUENOS ANIMAIS EM CURITIBA
}

\author{
Protocols used in veterinary clinics and hospitals for the control of endo and \\ ectoparasites in dogs and cats in Curitiba, PR, Brazil
}

\author{
BEIRÃO, B.C.B. ${ }^{1}$; ROSINELLI, A.S. ${ }^{1}$; Da SILVA, L.C.S. ${ }^{1}$; SANTOS, G.G.C. ${ }^{1}$; \\ RUTHES, L.D. ${ }^{1}$; MOLENTO, M.B. ${ }^{1}$
}

\author{
1 Universidade Federal do Paraná, Curitiba/PR. \\ Endereço para correspondência: Marcelo Beltrão Molento: molento@ufpr.br \\ RESUMO
}

Tendo-se em vista o alto risco zoonótico das doenças parasitárias e o crescente número de animais de companhia, potenciais transmissores, torna-se relevante o conhecimento especializado da área do controle parasitário. Na tentativa de controle sobre as parasitoses, os médicos veterinários assistiram ao desenvolvimento da resistência dos parasitos frente aos compostos químicos mesmo em pequenos animais, e isso se deve sobretudo à maneira como estas drogas foram utilizadas. $O$ clínico deve ter em mente que, além da saúde animal, deve ocupar-se da saúde humana, esclarecendo os proprietários quanto à problemática das zoonoses. Procurando ter um conceito acerca da atuação dos clínicos veterinários quanto a esse cenário, foram realizadas entrevistas em 120 estabelecimentos veterinários na cidade de Curitiba, PR, entre os meses de abril e maio de 2006. As perguntas envolviam o sexo e idade dos clínicos, 0 protocolo utilizado por estes contra as doenças parasitárias (principais princípios ativos recomendados pelos entrevistados, duração do tratamento, realização de exames coproparasitológicos para detecção de endoparasitas, raspado e inspeção de pele para verificação de ectoparasitas), parasitos mais comumente encontrados, eficácia e resistência dos antiparasitários e a comercialização destes. Constatou-se que 87,5 $\%$ dos entrevistados utilizam o protocolo profilático e supressivo para controlar e erradicar as parasitoses muitas vezes sem a devida exigência técnica, o que predispõe à resistência parasitária. Para se evitar tal quadro, para preservar a vida útil dos produtos e melhorar a qualidade de vida dos animais de companhia, os clínicos veterinários devem utilizar de forma consciente os compostos químicos, não abrindo mão do diagnóstico para as doenças parasitárias.

Palavras-chave: entrevistas; médicos veterinários; resistência parasitária; zoonoses

\begin{abstract}
The high risk for zoonotic diseases caused by animal parasites and the increasing numbers of pet animals, as infection vectors, it is relevant to understand the technical knowledge in the area of parasite control. The indication for parasite control has caused the development of parasite resistance and Veterinary clinicians have evidenced this against many compounds in small animals, this is due to the usage of preventive and suppressive treatment. Clinicians have to bear in mid that the animals' health may influence human health, mainly by zoonotic diseases. The objective of this work was to determine the opinion towards this situation through 120 interviews with Veterinarians from Curitiba, between April and May of 2006. The questions were related to the sex, age, and the clinical protocols against parasitic diseases (indicated compounds, treatment intervals, requested faecal exams, skin exams to determine ectoparasites), most prevalent parasites, efficacy, anthelmintic resistance, and the commerce of these drugs. It was found that $87.5 \%$ of the interviewers use the prophylactic and suppressive protocols to control or to eradicate parasites, mostly without technical criteria, predisposing the development of parasite resistance. Veterinary practitioners must recommend more laboratory diagnostics before prescribing anthelmintic drugs to avoid such a consequence, to prolong the lifespan of the anthelmintic and to improve pet animals quality of life.
\end{abstract}

Key words: interviews; parasite resistance; veterinary practitioners; zoonosis 


\section{INTRODUÇÃO}

O notável aumento no número de cães e gatos como animais de companhia e de guarda nos últimos anos coloca o Médico Veterinário em uma posição de destaque na orientação da população quanto aos diagnósticos, tratamento e controle das doenças nestes animais. Essas, além de representarem problemas à sociedade quando analisadas sob os aspectos do Bem-Estar animal e econômicos, representam também uma problemática dentro do contexto da saúde pública, como exemplo, o Toxocara sp. (Thompson e Roberts, 2001) e a larva migrans cutânea (LMC) (Lagaggio et al., 2005). A relação próxima e os diversos usos de animais de companhia, e mais sua distribuição, faz com que cães e gatos compartilhem com o homem, de forma indesejável, cerca de 60 espécies de parasitos, incluindo Toxocara sp., Ancylostoma sp., Echinococcus spp., Giardia sp., Cryptosporidium e Toxoplasma (Macpherson, 2005). Sendo assim, os Médicos Veterinários devem considerar as informações repassadas aos proprietários acerca da importância do manejo sanitário adequado dos animais e do ambiente (Harvey et al, 1991).

A negligência quase que completa acerca do conhecimento da biologia dos parasitos associada ao uso supressivo dos antiparasitários em animais de produção e de companhia levou ao fenômeno da resistência parasitária. Prichard et al. (1980) afirma que a resistência é uma característica herdada. $\mathrm{Na}$ ausência de tratamento, a freqüência do alelo de resistência é baixa. Segundo Molento (2004), a resistência é um resultado de um processo gradativo de seleção de indivíduos capazes de sobreviver após o constante emprego de um composto químico. A resistência não é uma conseqüência inevitável do uso de drogas, e sua seleção será dependente da capacidade reprodutiva conferida pelos alelos de resistência e susceptibilidade quando do uso de um medicamento.

O primeiro caso de resistência parasitária em bovinos foi documentado por Echevarria e Pinheiro (1990) citados por Souza et al. (2008) (albendazole e oxfendazole contra Haemonchus sp.). Em animais de companhia somente nos últimos anos começou-se a debater o tema (Thompson e Roberts, 2001). Parasitos de grande importância epidemiológica já foram relatados como resistentes em pequenos animais, frente ao palmoato de pirantel contra Toxocara canis (Dryden e Ridley, 1999) e Ancylostoma caninum (Jackson et al., 1987; Kopp et al., 2007) e a deltametrina contra Rhipicephalus sanguineus (Estrada-Pena, 2005).

De acordo com Molento (2001), devese, então, administrar a quantidade correta do composto, utilizar as drogas de maneira curativa, empregar bases químicas específicas contra a enfermidade, valer-se da droga antiparasitária de forma continuada, não fazendo a rotação até que se comprove 0 aparecimento da resistência e, ainda, o Médico Veterinário deve somente recomendar a combinação de drogas antiparasitárias com 0 conhecimento prévio da eficácia das drogas em questão (de no mínimo 95\%).

O objetivo do presente trabalho foi realizar um levantamento de protocolos utilizados por Médicos Veterinários em estabelecimentos veterinários situados na cidade de Curitiba. Procurou-se também estabelecer a relação entre a idade dos Médicos Veterinários e 0 padrão de resposta obtido; a prevalência dos parasitos, os princípios ativos utilizados, além do esquema e freqüência da profilaxia adotados.

\section{MATERIAL E MÉTODOS}

O município de Curitiba, capital do Estado do Paraná, situa-se na Região Sul 
do Brasil. Possui uma área de 432,418 km² (ParanaCidade, 2006) localizando-se no primeiro planalto, com altitude média de 930 m (Maack, 1981), latitude 2525'48" Sul e longitude 4916'15" Oeste. O clima é caracterizado como clima pluvial temperado-quente. As temperaturas médias variam de $21^{\circ} \mathrm{C}$, no verão a $13^{\circ} \mathrm{C}$, no inverno. A precipitação e a temperatura média anual são, respectivamente, $1.400 \mathrm{~mm}^{3}$ e $17^{\circ} \mathrm{C}$ (Maack,1981).

Para o presente estudo Curitiba foi subdividida em nove regionais, seguindo os critérios de localização das Ruas da Cidadania (sedes de administração regional da Prefeitura nos bairros), englobando os diferentes bairros contidos em cada regional.

Foi obtida uma listagem de endereços de todos os estabelecimentos veterinários do município de Curitiba concedida pelo Conselho Regional de Medicina Veterinária do Paraná. A partir destes dados verificou-se a proporção de estabelecimentos por região:

$P=(n / N) \times 100$, onde $P=$ proporção de estabelecimentos na região; $n=$ número de estabelecimentos na região e $\mathrm{N}=$ número total de estabelecimentos.

Após conferência da lista, constatouse a existência de empresas canceladas, duplicidade de registros e locais onde 0 veterinário foi apenas responsável técnico; esses registros foram descartados, obtendo-se assim o valor de $\mathrm{N}$ de 179.

Foram selecionados cem $(n=100)$ estabelecimentos do número total através de sorteio aleatório por regional. Tal sorteio consistiu na atribuição de letras às regionais, seguidas do número do estabelecimento existente na listagem concedida pelo CRMV-PR. Contudo, foram feitas vinte entrevistas a mais, que respeitaram as proporções regionais com o intuito de diminuir a margem de erro. Sendo assim a amostragem final totalizou 120 estabelecimentos veterinários. Este número foi obtido a partir da seguinte fórmula estatística (Zagury, 2006):

$$
d=t[(N-n) /(N-1)] ~ 1 / 2[(P . Q) / n] ~ 1 / 2,
$$
onde: $d=$ margem de erro $(\%) ; t=4,96$; $P . Q=2500 ; N=$ total da população (179); $\mathrm{n}=$ amostragem (120).

O questionário elaborado, contendo 19 questões, foi aplicado ao médico(a) veterinário(a) presente no estabelecimento, no período de abril a maio de 2006 . Diferentes entrevistadores visitaram cada estabelecimento, e um treinamento prévio foi feito de forma a padronizar a forma com que se deveria proceder durante as entrevistas. Dependendo da escolha do clínico o questionário poderia ser respondido na forma de uma entrevista ou até mesmo na ausência dos pesquisadores. O questionário incluía como informações particulares do entrevistado, a idade, sexo e região em que se localizou o estabelecimento.

Foram levantadas questões referentes à atitude clínica: principais medicamentos recomendados e parasitas encontrados, duração do tratamento, observação da falta de eficácia dos medicamentos, realização de exames coproparasitológicos para diagnóstico de endoparasitas, raspado e inspeção de pele para verificação de ectoparasitas, e a venda ou não de medicamentos no estabelecimento veterinário.

\section{RESULTADOS E DISCUSSÃO}

O trabalho foi realizado em todo o município de Curitiba, e alguns problemas ocorreram, como a impossibilidade de localizar estabelecimentos e veterinários que se recusaram ou que responderam de modo incompleto ao questionário. Quarenta entrevistados $(33,3 \%)$ não responderam a uma ou mais questões. Desta forma, foram acrescentados vinte questionários à amostra chegando a uma margem de erro de $9 \%$. Dos 120 entrevistados foi determinada a predominância do sexo feminino $(72,5 \%)$ como profissionais em medicina veterinária e uma idade média de 33,8 anos. 


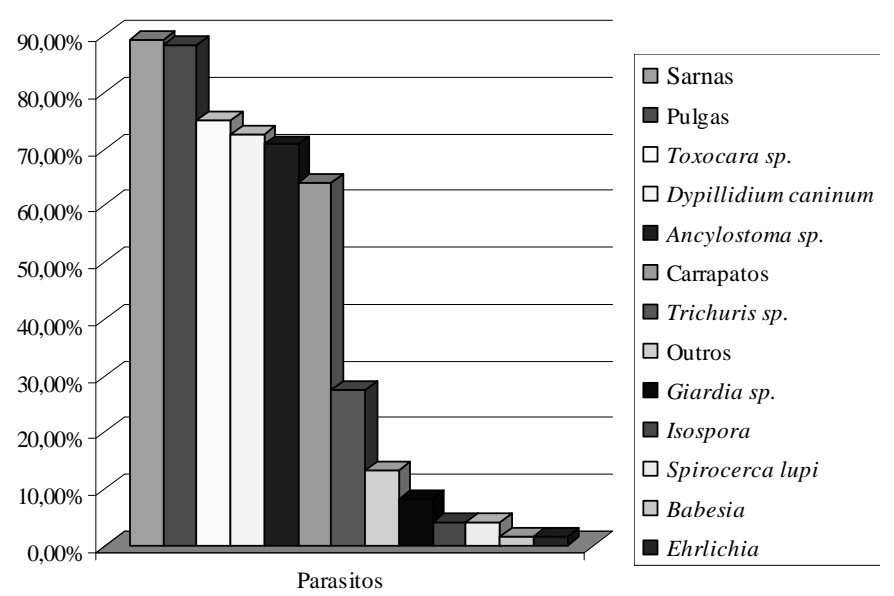

Gráfico 1 - Frequência do diagnóstico de parasitos em estabelecimentos veterinários em Curitiba.

Constatou-se que os profissionais valem-se, como meio de atualização, de revistas $(69,2 \%)$, trabalhos publicados $(68,3 \%)$, livros $(59,2 \%)$, Internet $(50,8 \%)$ e outros (12,5\%). Cunha et al. (2005) encontraram resultados semelhantes, em que $78,6 \%$ dos profissionais empregam como forma de atualização revistas, seguido por livros (75\%), trabalhos publicados $(67,8 \%)$, Internet $(46,4 \%)$ e outros $(10,7 \%)$. Há grande facilidade na obtenção de informação a respeito das diversas pesquisas que são desenvolvidas e rapidamente publicadas em todo 0 mundo. O uso de informes técnicos de indústrias farmacêuticas como base para decidir a postura clínica, como verificado na forma de publicação de trabalhos em separatas, reflete a natureza conservadora da profissão veterinária, e a relutância em aceitar novas pesquisas como sendo uma parte necessária da profissão (Cohen, 2000).

A maior percentagem de parasitismo observada clínica e laboratorialmente pelos entrevistados foi referente aos ectoparasitas como, sarnas $(89,2 \%)$ e pulgas $(88,3 \%)$ seguido dos endoparasitas, Toxocara sp. (75\%), Dipylidium caninum $(72,5 \%)$ e Ancylostoma sp. (70,8\%) (Gráfico 1), dado corroborado por Leite et al. (2004).

Várias metodologias de diagnóstico são passíveis de ser empregadas para as parasitoses. No entanto, é considerável o percentual de profissionais que nunca realiza exames parasitológicos $(24,2 \%)$ e os que apenas o fazem quando existe uma suspeita $(47,5 \%)$, na cidade de Curitiba. Cerca de $94 \%$ dos veterinários baseia sua indicação química somente através dos sinais clínicos do paciente para suspeitar de uma parasitose. Desses 17,5\% ignoram o histórico e outros dados relatados pelo proprietário para fazê-lo.

Os métodos mais utilizados para o exame parasitológico foram o raspado cutâneo $(65,8 \%)$ e a técnica de flutuação ou técnica de Willis (1921) (57,5\%). Os locais mais comumente utilizados para a realização das análises parasitológicas são os laboratórios veterinários $(60 \%)$ seguido da própria clínica (38,3\%).

Cunha et al. (2005) descreveram que $25 \%$ dos Médicos Veterinários em Santa Maria, RS já relataram a falta de eficácia para algum medicamento, sendo que no presente trabalho este valor foi de $38,3 \%$. A presença ou não de resistência foi um relato empírico fundamentado no critério clínico de cada entrevistado. Quando esse fenômeno ocorre, $67,5 \%$ dos clínicos em Curitiba mudam de princípio ativo, resultado similar ao encontrado em Santa Maria, onde a percentagem foi de $67,8 \%$. As outras condutas adotadas pelos veterinários em Curitiba frente ao problema da resistência é a indicação de compostos associados $(31,7 \%)$, aumento da dose $(3,3 \%)$ e outros $(9,2 \%)$. Salienta-se que 26 profissionais não responderam sobre 0 assunto. $\mathrm{O}$ aumento da dose administrada não é a indicação preconizada frente a resistência, já que não aumenta de modo significativo a quantidade de parasitos adultos eliminados. Segundo Molento (2001), o uso combinado de diferentes grupos químicos em uma droga para o combate da resistência parasitária em cães expõe animais sadios a níveis químicos tóxicos. Assim, é de grande importância encontrar meios para utilizar as substâncias anti-helmínticas de modo 


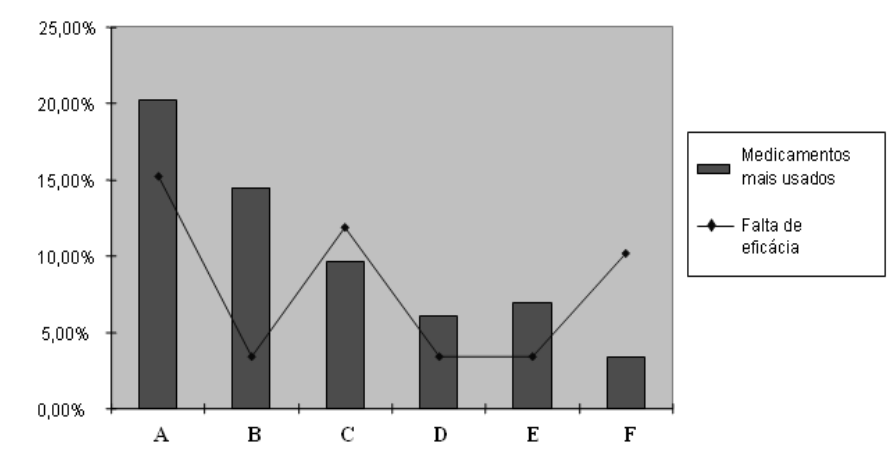

$\mathrm{A}=$ Pirantel; $\mathrm{B}=$ Febantel + Praziquantel + Ivermectina + Pirantel; $C=$ Pirantel + Praziquantel; $D=$ Milbemicina Oxima + Praziquantel; $\mathrm{E}=$ Fipronil; $\mathrm{F}=$ Ivermectina

Gráfico 2 - Relação entre os princípios ativos empregados por clínicos veterinários de Curitiba para o tratamento das endo e ecto parasitoses e suas correspondentes faltas de eficácia, conforme afirmado em questionário respondido de abril a maio de 2006.

sustentável, preservando sua eficácia por um tempo máximo e em tantas espécies de parasitas quanto possível (Von Samson-Himmelstjerna e Blackhall, 2005).

Foi constatado que $79,2 \%$ dos médicos veterinários em Curitiba comercializam medicamentos antiparasitários em seus estabelecimentos. Cunha et al. (2005) verificaram que $46,5 \%$ dos entrevistados comercializaram medicamentos no local de atendimento. Este dado pode eventualmente denotar a influência do mercado sobre a tomada de decisão do profissional acerca de seus protocolos terapêuticos.

Os princípios ativos mais empregados pelos clínicos são, de modo geral, aqueles que empiricamente apresentam menor eficácia, fato este devido ao esquema de tratamento em que são utilizados (Gráfico 2). Foi relatada uma baixa eficácia da ivermectina (principalmente contra sarnas), demonstrando sua ação inespecífica contra esse parasita. Isso se deve também ao fato de que esse composto já foi utilizado intensivamente no passado.

Em estudo realizado na Alemanha, Reino Unido e Canadá foi demonstrada a falta de eficácia do princípio ativo pamoato de pirantel contra Toxocara canis, Toxascaris leonina, Ancylostoma caninum e Uncinaria stenocephal em doses baixas. Já em doses superiores os autores observaram a eficácia do produto apenas contra Toxascaris leonina e Ancylostoma caninum (Clark et al., 1991). Segundo Epe e Daugschies (1995) os compostos químicos pirantel e praziquantel são os compostos mais utilizados em cães na região de Niedersachsen, Alemanha. Waller (2006) observou que a causa do sucesso dos anti-helmínticos modernos, com notáveis propriedades de redução da carga parasitária, espectro de ação, segurança, facilidade de uso e baixo custo, foi muito responsável pela queda na sua eficácia.

Dentre os médicos veterinários entrevistados, 30,8\% declararam aceitar sugestões dos proprietários no calendário profilático, resultado este semelhante ao valor de $32 \%$ encontrado por Cunha et al. (2005). Grande parte dos $67,5 \%$ que declararam não aceitar sugestões do proprietário, em Curitiba, justificou tal conduta por se considerarem tecnicamente mais aptos na escolha e decisão acerca do calendário profilático.

Com relação à atitude tomada frente às endo ou ectoparasitoses, o presente estudo constatou a predominância do esquema profilático $(87,5 \%)$ com uma dose inicial e sua posterior repetição em quinze dias $(59,2 \%)$ e frequência de tratamento profilático de 90 em 90 dias $(57,5 \%)$. Os estudos que abordam a resistência parasitárias em cães e gatos são recentes, o que indica a necessidade de uma constante atualização, mesmo para a geração mais nova de entrevistados (21-30 anos) (Gráfico 3). Todas as faixas etárias utilizam o esquema profilático de tratamento contra parasitoses, que é tido como um dos fatores que levam ao fenômeno da resistência. O potencial de seleção depende da dinâmica populacional do hospedeiro e dos parasitas além dos efeitos epidemiológicos do tratamento aplicado. Se for permitido, o desenvolvimento de uma população de parasitas 


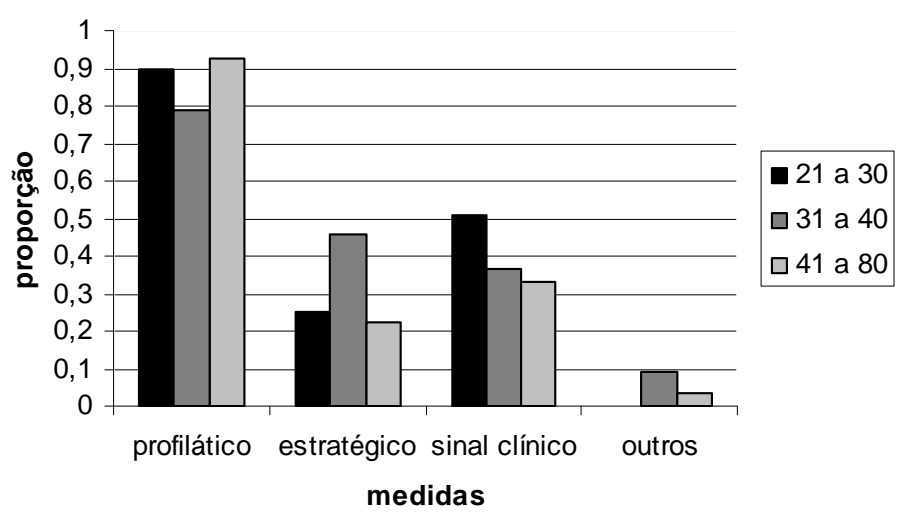

Gráfico 3 - Proporção entre as distintas formas de tratamento por intervalo de idade dos médicos veterinários.

resistentes em um grupo isolado de animais, através de um esquema profilático e supressivo de tratamento, qualquer animal retirado deste grupo e posto em um local livre de ovos ou larvas de parasitas iria desenvolver uma comunidade parasitária destacada, a qual seria inteiramente resistente à quimioterapia. Isto seria intensificado se o animal fosse tratado imediatamente antes de ser realocado. Este efeito, chamado "efeito fundador", poderia ser diluído em um ambiente que já tivesse larvas de vida livre as quais dissolveriam a população resistente (Thompson e Roberts, 2001).

Bossard et al. (1998) postularam que a ecologia dos genes de resistência para pulgas provavelmente envolve a transferência do parasito entre hospedeiros, movimento do hospedeiro, refugia, "efeito fundador", e a mortalidade por efeitos abióticos. A liberação constante de inseticida, promovida por colares antipulga e resíduos com meias-vidas longas podem ser responsáveis pelo desenvolvimento de resistência, assim como a aplicação profilática de antiparasitários.

Evidenciou-se que os protocolos terapêuticos mais utilizados por clínicos no município de Curitiba são profiláticos, 0 que, no controle das doenças parasitárias, pode significar 0 aparecimento de resistência aos compostos utilizados (Molento, 2004). Verificou-se uma alta taxa de efetuação de reconsulta (95\%), as quais deveriam ser empregadas para a discussão e elucidação acerca das zoonoses. Em estudo conduzido por McGlade et al. (2003) com proprietários de felinos na cidade de Perth, Austrália, foram aplicados questionários sobre possíveis zoonoses transmitidas por parasitos gastrintestinais de felinos e, embora a maioria $(64,5 \%)$ dos proprietários estivesse ciente do risco de zoonoses, menos da metade $(42,8 \%)$ sabia quais os seus meios de transmissão para humanos. $O$ interesse em controlar as doenças passa pelo entendimento de que não existe medicamento $100 \%$ eficaz em todas as aplicações e contra a totalidade dos organismos presentes.

\section{CONCLUSÕES}

Os protocolos terapêuticos mais utilizados por clínicos no município de Curitiba são profiláticos, o que, no controle das doenças parasitárias, pode significar o aparecimento de resistência aos compostos utilizados.

Verificou-se alta taxa de efetuação de reconsulta (95\%), as quais deveriam ser empregadas para a discussão e elucidação acerca das zoonoses.

\section{REFERÊNCIAS}

BOSSARD, R.L.; HINKLE, N.C.; RUST, M.K. Review of insecticide resistance in cat fleas (Siphonaptera: Pulicidae). Journal of Medical Entomology, v.35, n.4, p.415-422, 1998.

CLARK, J.N.; DAURIO, C.P.; BARTH, D.W. et al. Evaluation of a beef-based chewable formulation of pyrantel pamoate against induced and natural infections of hookworms and ascarids in dogs. Veterinary Parasitology, v. 40, n.1-2, p. 127-133, 1991.

COHEN, M.L. Changing patterns of infectious disease. Nature, v.406, n.6797, p.762-767, 2000.

CUNHA, M.; GAVIÃO, A.; BERTOLETTI, B. et al. Levantamento de protocolos utilizados em clínicas veterinárias para controle de endo e ectoparasitas 
em pequenos animais em Santa Maria, RS. Salão de Iniciação Científica e Mostra Científica, 5., Pontifícia Universidade Católica do Rio Grande do Sul, Outubro, 2005.

DRYDEN, M.W.; RIDLEY, R.K. Efficacy of fenbendazole granules and pyrantel pamoate suspension against Toxocara canis in greyhounds housed in contaminated runs. Veterinary Parasitology, v.82, n.4, p.311-315, 1999.

EPE. C.; DAUGSCHIES, A. Inquiry of veterinarians in Niedersachsen concerning the occurrence of parasitic diseases and their control in small animals. Dtsch Tierarztl Wochenschr, v.102, n.2, p.78-80, 1995.

ESTRADA-PENA, A. An appraisal of the resistance status against acaricides by the brown dog tick, Rhipicephalus sanguineus. Revue de Médecine Vétérinaire, v.156, n.2, p.67-69, 2005.

HARVEY, J.B.; ROBERTS, J.M.; SCHANTZ, P.M. Survey of veterinarians' recommendations for treatment and control of intestinal parasites in dogs: public health implications. Journal of the American Veterinary Medical Association, v.199, n.6, p.702707, 1991.

JACKSON R.; LANCE D.; TOWNSEND K. et al. Isolation of anthelmintic resistant Ancylostoma caninum. New Zealand Veterinary Journal, v.35, n.12, p.215-216, 1987.

KOPP, S.R.; KOTZE, A.C.; MCCARTHYM, J.S. et al. High-level pyrantel resistance in the hookworm Ancylostoma caninum. Veterinary Parasitology, v.143, n.3-4, p.299-304, 2007.

LAGAGGIO, V.R.A.; NETO, L.M.S.; LAGAGGIO ,R.A. et al. Cães abandonados versus contaminação ambiental . Revista de Patologia Tropical, v.34, Suplemento Especial, 2005.

LEITE, L.C.; MARINONI L.P.; CÍRIO, S.M. et al. Endoparasitas em cães (Canis familiaris) na cidade de Curitiba-Paraná-Brasil. Archives of Veterinary Science v.9, n.2, p.95-99, 2004.

MAACK , R. Geografia física do Estado do Paraná. 2.ed. Rio de Janeiro: Livraria José Olympio Editora, 1981. 442p.
MacPHERSON, C.N. Human behaviour and the epidemiology of parasitic zoonoses. International Journal of Parasitology, v.35, n.11-12, p.13191331, 2005.

MCGLADE, T.R.; ROBERTSON, I.D.; ELLIOT, A.D. et al.. Gastrointestinal parasites of domestic cats in Perth, Western Australia. Veterinary Parasitology, v.117, p.251-262, 2003.

MOLENTO, M.B. Opções de tratamento e risco de resistência. DBO Saúde Animal. Outubro, p.18-22, 2004.

MOLENTO, M.B. Resistência parasitária: a indústria na contramão Revista Clínica Veterinária, n.32, p.10, 2001.

PRICHARD, R.K.; HALL, C.A.; KELLY, J.D.; MARTIN, I.C.; DONALD, A.D. The problem of anthelmintic resistance in nematodes. Australian Veterinary Journal, n.5, v.56, p.239-251, 1980.

SERVIÇO SOCIAL AUTÔNOMO PARANACIDADE. Disponível em: http://www.paranacidade.org.br/mun icipios/municipios.php> Acesso em: 08 jan. 2008.

SOUZA, A.P.; RAMOS, C.I.; BELLATO, V. et al. Resistência de helmintos gastrintestinais de bovinos a anti-helmínticos no Planalto Catarinense. Ciência Rural, v.38, n.5, p.1363-1367, 2008.

THOMPSON, R.C.A.; ROBERTS, M.G. Does pet helminth prophylaxis increase the rate of selection for drug resistance? Trends in Parasitology, v.17, n.12, p.576-578, 2001.

VON SAMSON-HIMMELSTJERNA, G.; BLACKHALL, W. Will technology provide solutions for drug resistance in veterinary helminths? Veterinary Parasitology, v.132, p. 223-239, 2005.

WALLER, P.J. From discovery to development: Current industry perspectives for the development of novel methods of helminth control in livestock. Veterinary Parasitology, v.139, p.1-14, 2006.

WILLIS, H.H. A simple levitation method for detection of hookworm ova. Medical Journal of Australia, North Sidney, v.8, p.375-376, 1921.

ZAGURY, T. O professor refém. Rio de Janeiro, Editora Record, 2006. 302p. 